\title{
Automated versus Manual Blood Pressure Measurement: A Randomised Crossover Trial
}

\author{
Megan Heinemann, RN, BN (Hons) \\ Registered Nurse (Division 1) \\ LaTrobe Regional Hospital, Traralgon \\ Victoria. Australia.
}

Ken Sellick, RN, PhD

Senior Research Fellow, School of Nursing \& Midwifery, Monash University, Australia

Claire Rickard, RN, PhD, GradDip (Crit Care)

Professor of Nursing

School of Nursing \& Midwifery and the Research Centre for Clinical and Community Practice Innovation

Griffith University, Australia

Pam Reynolds, RN, BN, GradDip (Crit Care)

Associate Lecturer/Clinical Coordinator

School of Health Sciences, Nursing \& Midwifery

RMIT University, Australia.

Matthew McGrail, BSc (Hons), GradDipIT

Lecturer

Department of Rural \& Indigenous Health,

Monash University, Australia

Correspondence: $\quad$ Dr Ken Sellick, School of Nursing \& Midwifery, Monash University, Northway's Road, Churchill, Victoria 3842, Australia

Email:ken.sellick@med.monash.edu.au 


\begin{abstract}
This study evaluated the accuracy and reliability of the Dinamap 8100 automated blood pressure machine against three internationally recognized criteria. Systolic and diastolic blood pressures were taken concurrently by two nurses using the automated machine and a manual sphygmomanometer. Results demonstrated agreement between automated and manual readings on one set of criteria for both systolic and diastolic pressures, and support for systolic readings only on one other criterion. Comparison of mean differences between automated and manual measures showed the automated machine consistently under-read both systolic and diastolic blood pressures. The conclusion from this study was that the Dinamap 8100 machine can be used with some degree of confidence to assess systolic blood pressures in a general population of adult hospital in-patients, but with caution when taking diastolic readings.
\end{abstract}

Keywords: automated blood pressure machines; blood pressure; measurement; nursing practice; vital signs; 


\section{INTRODUCTION}

A routine aspect of nursing practice is obtaining accurate and reliable assessments of patients' blood pressure in order to ensure optimal and appropriate care, and avoid subjecting patients to unnecessary risk ${ }^{1}$. Traditionally, blood pressures have been measured using a manual mercury sphygmomanometer, but in recent years the use of automated blood pressure machines such as the Dinamap and Omron have been the trend in most hospitals. However, many nurses question the accuracy and reliability of these machines when used in the clinical setting, and still prefer the manual sphygmomanometer which is considered the 'gold standard' when used by a trained observer $^{2}$.

There is considerable debate in the literature on the accuracy of automated blood pressures machines. The general consensus in that automated machines are inaccurate and unreliable ${ }^{3,4,5}$ : a situation that can result in inappropriate administration of medications, withholding treatment unnecessarily or other adverse clinical consequences. However, the level of agreement between automated and manual blood pressure measurements can vary for systolic and diastolic readings, and in relation to type of machine and patient population. Prior to the publication of the British Hypertensive Society (BHS) protocol ${ }^{2}$ and the Association for the Advancement of Medical Instrumentation (AAMI) standards ${ }^{6}$, internationally recognized criteria for determining the accuracy and reliability of automated blood pressure devices were not available. The BHS protocol requires the automated device be compared with blood pressures measured by trained observers using a mercury sphygmomanometer and stethoscope. The protocol also specifies methodological requirements such as training observers, blood pressure measurement procedures, instrument calibration, and data analyses. 


\section{LITERATURE REVIEW}

A review of the literature located six well designed studies that used established criteria to determine the accuracy and reliability of blood pressure measurements taken with an automated device. The majority of these studies evaluated the Dinamap 8100 machine, and all but two used BHS and AAMI criteria to establish level of equivalence. The main differences between the studies were the age and health status of participants. Three studies included children; of which two were well populations ${ }^{7,8}$ and the other children with diabetes ${ }^{9}$. Three studies recruited adult patient samples: one attending a pre-operative day surgery clinic ${ }^{10}$, one an emergency department ${ }^{11}$, and the other pregnant women in a hospital maternity unit ${ }^{12}$. Studies also varied in sample size, number and sequence of measures, and level of control for cuff size and interrater reliability.

Findings from these studies tend to confirm the opinion of many nurses that automated blood pressure devices are unreliable. Five studies reported that the Dinamap 8100 machine consistently over-read systolic and under-read diastolic blood pressures $7,8,9,10,11$ and one study, using the Welch Allyn 'Vital Signs' monitor, under-read both systolic and diastolic pressures ${ }^{12}$. Differences between devices ranged from $2.6 \mathrm{mmHg}$ to $11 \mathrm{mmHg}$ for systolic and $1.5 \mathrm{mmHg}$ to $5.5 \mathrm{mmHg}$ for diastolic readings. Some of these differences were considered to be clinically significant. The researchers' recommendations were that an automated machine is acceptable for use in pre-pubertal children with diabetes ${ }^{9}$, but not for children in a clinical setting where an accurate absolute measure is required ${ }^{8}$; is suitable for normotensive patients but not for patients with hypertension 10,11 ; and when used with pre-eclampsia women readings are to be interpreted with caution ${ }^{12}$. 
It is clear from the review that further studies are needed to establish the accuracy and reliability of automated blood pressure machines for use within the clinical setting, particularly with adult patients presenting with a variety of clinical conditions. These studies need to employ rigorous research designs to control for the many factors that can affect blood pressure readings, and use established criteria for determining the equivalence between automated machines and the recognized 'gold standard', namely the manual mercury sphygmomanometer. Of note is that no studies tested the use of automated blood pressure machines with adult hospital patients presenting with common illnesses and health concerns.

The aim of the current study was to test the accuracy and reliability of measurements taken with the Dinamap 8100 automated blood pressure machine using a sample of hospital in-patients presenting with common medical and surgical conditions. To answer the research question, a test of the null hypothesis was proposed which stated that there is no significant difference in systolic and diastolic blood pressure readings taken with a Dinamap 8100 machine and a manual mercury sphygmomanometer.

\section{METHOD}

\section{Design}

A randomised crossover clinical trial design was used to provide a rigorous test of the hypotheses under investigation. Blood pressure measurements for each participant were taken concurrently by two nurses using a manual and automated blood pressure device with the type of device, arm and the nurse taking the measurements randomly determined. The two nurses remained blind to each other's recordings. Approval to conduct the study was obtained from the Hospital Ethics Committee. 


\section{Sample}

A convenience sample of patients was recruited from a medical ward, general medical- surgical ward, orthopaedic ward and the critical care unit of a large regional hospital in rural Victoria, Australia. The criteria for selecting patients into the study were the patient was clinically stable and lucid (as determined by the RN caring for them), English speaking and over 18 years of age. Patients suffering acute pain or confusion, premedicated for surgery, or had limb amputation or lymphatic damage to the limbs were excluded to eliminate factors that could effect bilateral blood pressure measurements.

A power calculation determined a sample size of 73 was required to achieve a power estimate of 0.8 for paired samples to detect a mean difference between measurement techniques of 5 $\mathrm{mmHg}(\mathrm{SD} 15 \mathrm{mmHg}$ ) with a Type 1 error rate set at 0.05 . Of 138 patients who met the selection criteria 63 consented to participate, providing a sample size slightly below the number required.

\section{Blood pressure measurement}

\section{Equipment}

The manual device used was a standard mercury sphygmomanometer with an Accoson cuff and Littman Classic II stethoscope. The automated device was a Dinamap 8100 machine (Critikon, Tampa PL, Tampa) with dura - cuf cuffs. All machines used in the study were calibrated and checked for compliance to machine standards by the Hospital's Biomedical Engineering Department. 


\section{Procedure}

Blood pressure measurements were taken by two nurses trained in the use of both devices: one an experienced registered nurse with an intensive care nursing qualification and the other a recently qualified nurse. Measurements were obtained using standardized procedures for each device $^{4}$.

\section{Preliminary preparation}

- Obtain informed written consent from patient

- Position machines on the appropriate side of the bed.

- Patient positioned supine, with arms straight and legs uncrossed.

- Determine cuff size by measuring arm circumference at the halfway point between shoulder tip and elbow tip. Select appropriate cuff size using the American Heart Association Guidelines ${ }^{13}$.

- Fit cuff to the patient's arm.

- Face machine dial away from the other nurse.

\section{Mercury sphygmomanometer}

- Determine a 'systolic estimate' of the patient's blood pressure by inflating the cuff until the radial pulse can no longer be palpated. Inflate cuff a further $30 \mathrm{mmHg}$ and release the valve at $2 \mathrm{mmHg}$ per second until the radial pulse reappears. Record this reading as the systolic blood pressure estimate.

- Wait 3 minutes to allow adequate circulation to return. 
- Inflate cuff to $30 \mathrm{mmHg}$ higher than the 'systolic estimate'. Slowly deflate the cuff at $2 \mathrm{mmHg}$ per second and, using a stethoscope, record measures corresponding to the $1^{\text {st }}$ and $5^{\text {th }}$ Korotkoff sounds.

- Record systolic and diastolic measures on data collection form.

\section{Dinamap machine,}

- Plug machine into power point.

- Press 'power' button on machine.

- Press 'start' button to commence blood pressure measurement.

- Record displayed systolic and diastolic readings on data collection form.

\section{Data collection}

A data collection form was designed to record systolic and diastolic blood pressure readings, type of device, cuff size, arm used, and the date and time blood pressure measurements were taken. Also noted on the form were patient ID number, age, gender, weight, diagnosis, if the patient had a history of cardiac or renal disease and any comments on the procedure.

\section{Randomisation}

Two data collection forms for each patient were pre-prepared to randomise blood pressure device sequence (auto then manual/manual then auto) and arm (right/left) for each nurse. Eighty paired data forms covering all permutations were printed and placed in a sealed envelope. Before taking the patient's blood pressure, one of the nurses randomly selected an envelope containing the forms. 


\section{Pilot Study}

A pilot study was conducted to test blood pressure measurement procedures, and to ensure the design requirements of randomisation and blind methodology could be achieved. The pilot study also enabled the nurses to perfect their blood pressure measurement technique and achieve close agreement on blood pressure readings.

The two nurses jointly obtained manual blood pressure recordings from 20 staff volunteers using a sphygmomanometer and a double-ear piece stethoscope. Results showed only minimal variation in readings for both systolic and diastolic blood pressures. The full procedure using the manual and automated devices was then trialed on a sample of 24 staff volunteers. Positioning the volunteer was easily achieved and no problems were experienced with the change over procedure.

\section{Data Analysis}

All information from the data collection forms were entered into Microsoft Excel and then imported into Stata 8.2 and SPSS (Version 14.0) for analysis. A series of descriptive analyses (frequency distributions, summary statistics and correlations) were conducted to describe sample characteristics and key measures, and to examine inter-rater reliability and the relationships between automated and manual blood pressure measurements.

The procedure proposed by Jin et al ${ }^{9}$ was used to determine the level of agreement between the two devices for measuring blood pressures. This procedure examines three sets of criteria: AAMI criteria ${ }^{6}$, the BHS grading system ${ }^{1,2}$; and examination of Bland-Altman plots ${ }^{14}$. The AAMI method specifies a mean difference between devices of $<5 \mathrm{mmHg}$ with a standard deviation of $\leq 8 \mathrm{mmHg}$. The BHS criteria is based on the cumulative percentage of mean differences between the two devices for scores of $\leq 5 \mathrm{mmHg}, \leq 10 \mathrm{mmHg}$ and $\leq 15 \mathrm{mmHg}$ 
categorized into four grades (see Table 1). A grade of A or B indicates agreement between the two devices.

\section{INSERT TABLE 1 ABOUT HERE}

The Bland-Altman method plots the mean differences in blood pressure measurements between the two devices against the average for both devices, along with regression lines and $95 \%$ confidence limits. The criteria for agreement are a horizontal regression line ( $\mathrm{r}^{2}$ close to zero) and narrow $95 \%$ confidence limits.

\section{RESULTS}

\section{Sample Characteristics}

Examination of sample characteristics showed a similar proportion of male (49.2\%) and female $(50.8 \%)$ patients and an age range of 19 to 93 years (mean age $=66.36)$. Reasons for admission to hospital included general and orthopaedic surgery, respiratory illnesses, abdominal and cardiac conditions and chest pain. The most common reasons for admission were orthopaedic surgery (32\%) and respiratory illnesses (16\%). Of the 63 patients included in the study, 64\% had a history of cardiac illness and $9.5 \%$ reported a history of renal disease

\section{Inter-rater Reliabilty}

Pearson correlation coefficients were calculated to determine the inter-rater reliability of systolic and diastolic readings taken by the two nurses using both devices. Results found significant positive correlations on all four measures (range .64 to .79), indicating high agreement 
between the two nurses. Level of agreement on automated diastolic measures was notably lower $(\mathrm{r}=.64)$ than the other three measures.

\section{Agreement between Manual and Automated Devices}

\section{AAMI Criteria}

Table 2: shows comparative means, standard deviations and difference scores for automated and manual measurements of systolic and diastolic blood pressures. Paired t-test analyses found automated systolic and diastolic readings were significantly lower than those taken manually. When mean difference scores and standard deviations were examined against AAMI criteria (mean difference $<5 \mathrm{mmHg}$, standard deviation $\leq 8 \mathrm{mmHg}$ ), agreement between the two devices was confirmed for systolic readings only.

\section{INSERT TABLE 2 ABOUT HERE}

\section{BHS Criteria}

BHS grades for automated and manual blood pressures (see Table 4) show the criteria for equivalence (Grade A or B) was not achieved for both systolic and diastolic readings.

\section{INSERT TABLE 3 ABOUT HERE}




\section{Bland and Altman Plots}

Figures 1 to 2 present the Bland-Altman plots of blood pressure differences (manualautomated) against the mean averages for both devices for systolic and diastolic readings. Also included in the graphs are the regression lines and 95\% CI limits. Results show regression lines were flat ( $\mathrm{r}^{2}$ close to zero) and confidence limits reasonably narrow for both systolic and diastolic readings.

\section{INSERT FIGURES 1 AND 2 ABOUT HERE}

\section{DISCUSSION}

The aim of this study was to establish the equivalence of blood pressure measurements taken with an automated machine (Dinamap 8100) to those obtained using a manual mercury sphygmomanometer. A key feature of the study was that blood pressure measurements were obtained from a general sample of adult hospitalized patients heterogeneous for age, gender and diagnosis.

Three sets of criteria were used to test the hypothesis of "no difference" between Dinamap and manual blood pressure measurements: AAMI criteria, BHS grades and Bland-Altman plots. Findings provided only partial support for the hypothesis, with agreement between devices on Bland-Altman criteria for both systolic and diastolic blood pressures, and on the AAMI criteria for systolic readings only. The BHS criterion for device equivalence was not achieved for either systolic or diastolic measurements. Thus the conclusion is that the Dinamap 8100 machine can be used with some degree of confidence when assessing the systolic blood pressure of hospital 
in-patients presenting with a range of medical and surgical conditions, but caution is needed when used to take diastolic measures.

In the present study readings on the Dinamap machine were significantly lower for both systolic and diastolic blood pressures. These findings support the study by Reinders et al ${ }^{12}$ which reported lower systolic and diastolic reading with the Dinamap in pre-eclamptic women. These differences were attributed to haemodynamic changes associated with pre-eclampsia, such as a decrease in arterial compliance and intravascular volume, which could result in an incorrect reading at a given pressure. The tendency of the Dinamap to under-read diastolic blood pressure was also reported by the other five studies, irrespective of whether readings were taken from well

children $^{7,8}$, children with diabetes ${ }^{9}$, or adult patients attending a hospital emergency department ${ }^{11}$ or day surgery clinic ${ }^{10}$.

\section{Implications for nursing practice}

In recent years the use of automated blood pressure machines has become the norm in most hospitals. Reasons for this trend are that automated machines are easier to use, allow continuous or intermittent blood pressure monitoring, provide printouts of readings, and for some machines, allow pulse rate and oxygen saturation levels to be taken concurrently. The main limitations, on the other hand, are the potential to provide false readings, especially on the first inflation, patient discomfort, and the need to regularly calibrate the machine ${ }^{7}$. It is important nurses are aware that automated blood pressure machines can provide less reliable readings than those taken with a manual sphygmomanometer. The cardinal rule is when the nurse is unsure about the accuracy of the automated readings; the blood pressure is taken manually and checked by an experienced colleague. 
An important question is whether an automated blood pressure device can be recommended for use in the clinical setting. The conclusion from this study is that the Dinamap 8100 machine can be used with some degree of confidence to assess the systolic blood pressure of general hospital in-patients but with less confidence when taking diastolic readings. Although Jin et al ${ }^{9}$ found the Dinamap acceptable for use in clinical situations, others had some reservations. For example, Coe \& Houghton ${ }^{10}$ concluded that the Dinamap is suitable for normotensive adults but not for patients with high or low blood pressure, whereas Reinders et al ${ }^{12}$ considered the Dinamap acceptable for women with a normal pregnancy but not for those with pre-eclampsia. Barker $e t$ $a l^{8}$ and Wattigney et $a l^{7}$, on the other hand, are more hesitant in their opinion based on a number of study limitations.

\section{Concluding Statement}

Nurses have long doubted the accuracy and reliability of automated blood pressure machines. This view is consistent with the personal experiences of the researchers, anecdotal comments by experienced clinicians, and equivocal evidence reported in the research literature. All six studies reviewed used strong research designs and recognised criteria for determining equivalence between automated and manual blood pressure devices. Therefore, the most likely explanation for the variation in research findings is the populations from which blood pressure measures were obtained. Of note is the high proportion of studies with children, and relatively few that used adult in-patient hospital samples. Hence one recommendation for further investigation is to replicate the present study using a similar patient population paying particular to attention to statistical power, inter-rater reliability and control over procedural aspects which can potentially bias results. In order to allay nurses' concerns about the accuracy of automated blood pressure devices, further studies are needed across a range of clinical populations and 
settings. Only then will nurses feel confident they are using accurate and reliable blood pressure measurements as the basis for clinical decision-making.

\section{Acknowledgments}

The authors wish to thank the nursing staff and patients from the participating hospital who made this study possible.

\section{References}

1. Little P, Barnett J, Barnsley L, Marjoram, J, Fitzgerald-Barron A, Mant, D.Comparison of acceptability of and preferences for different methods of measuring blood pressure in primary care. British Medical Journal 2002; 325: 258-259.

2. O’Brien E, Petrie J, Littler W, de Swiet M., Padfield P, Altman, D. et al. The British Hypertension Society protocol for the evaluation of blood pressure measuring devices. Journal of Hypertension 1993; 11 (Suppl 2): S43-S62.

3. Carney S. L, Gillies A H, Green S L, Paterson O, Taylor M. S, Smith A J. Hospital blood pressure measurement: staff and device assessment. Journal of Quality in Clinical Practice 1999; 19: 95-98.

4. Crisp J, Taylor C. Potter and Perry’s Fundamentals of Nursing. Sydney: Mosby, 2001. 
5. O’Brien E, Waeber B, Parati G, Staessen J, Myers M G. Blood pressure monitoring devices: Recommendations of the European Society of Hypertension. British Medical Journal 2001; 322: $531-536$.

6. White W B, Berson A S, Robbins C. et al. National standard for measurement of resting ambulatory blood pressure with automated sphygmomanometers. Hypertension 1993; 21: 504509

7. Wattigney W A, Webber L S, Lawrence M D, Berenson G S. Utility of an automatic instrument for blood pressure measurement in children: The Bogalusa heart study. American Journal of Hypertension 1996; 9: 256-262.

8. Barker M E, Shiell A W, Law C M. Evaluation of the Dinamap 8100 and Omron M1 blood pressure monitors for use in children. Paediatric and Perinatal Epidemiology 2000; 14: 179-186.

9. Jin R Z, Donaghue K C, Fairchild J M, Chan A, Silink M. Comparison of Dinamap 8100 with sphygmomanometer blood pressure measurement in a prepubertal diabetes cohort. Journal of Paediatric Child Health 2001; 37: 545-549.

10. Coe T R, Houghton K. Comparison of the automated Dinamap blood pressure monitor with the mercury sphygmomanometer for detecting hypertension in the day case pre-assessment clinic. Ambulatory Surgery 2002 ; 10: 9-15. 
11. Chong W F, Tan C H P, Yeo S L R, Ching S S, Cheng L. A study on reliability and accuracy of digital and manual sphygmomanometers. Singapore Nursing Journal 2000; 27: 28-35.

12. Reinders A, Cuckson A C, Jones C R, Poet R, O'Sullivan G, Shennan A H. Validation of the Welch Allyn 'Vital Signs' blood pressure measurement device in pregnancy and pre-eclampsia. British Journal of Obstetrics and Gynaecology 2003; 110: 134-138.

13. Perloff D, Grim C, Flack J, Frohlich E, Hill M, McDonald M, Morgenstern B. Human blood pressure determination by sphygmomanometry. Circulation 1993: 88; 2460-2467.

14. Bland J M, Altman D G. Statistical methods for assessing agreement between two methods of clinical measurement. Lancet 1986; 1:307-310. 
Table 1 British Hypertension Society criteria

\begin{tabular}{lccc}
\hline Grade & $\begin{array}{c}\text { Cumulative percentage of absolute difference } \\
\mathbf{5 5}\end{array}$ & $\mathbf{5 1 0}$ & $\mathbf{5 1 5}$ \\
\hline Grade A (very good) & 60 & 85 & 95 \\
Grade B (good) & 50 & 75 & 90 \\
Grade C (poor) & 40 & 65 & 85 \\
Grade D (very poor) & & worse than C \\
\hline
\end{tabular}

Table 2 Means, standard deviations, $\mathrm{t}$ values and mean difference scores for manual and automated systolic and diastolic blood pressures.

\begin{tabular}{llllll}
\hline & Manual & Automated & Paired t-test & \multicolumn{2}{l}{ Difference scores } \\
& Mean (SD) & Mean (SD) & p-value & Mean (SD) & $(95 \% \mathrm{CI})$ \\
\hline Systolic & $128.47(17.4)$ & $125.34(17.8)$ & 0.005 & $3.1312 .2)$ & $(0.97-5.28)$ \\
Diastolic & $73.06(10.66)$ & $67.81(12.25)$ & $<0.001$ & $5.22(10.4)$ & $(3.38-7.07)$ \\
\hline
\end{tabular}

Table 3 BHS grades for systolic and diastolic blood pressure readings

\begin{tabular}{lcccc}
\hline & $\mathbf{5}$ & $\mathbf{5 1 0}$ & $\mathbf{5 1 5}$ & Grade \\
\hline Systolic & $44 \%$ & $67 \%$ & $80 \%$ & $\mathrm{C}$ \\
\hline Diastolic & $37 \%$ & $69 \%$ & $85 \%$ & $\mathrm{D}$ \\
\hline
\end{tabular}




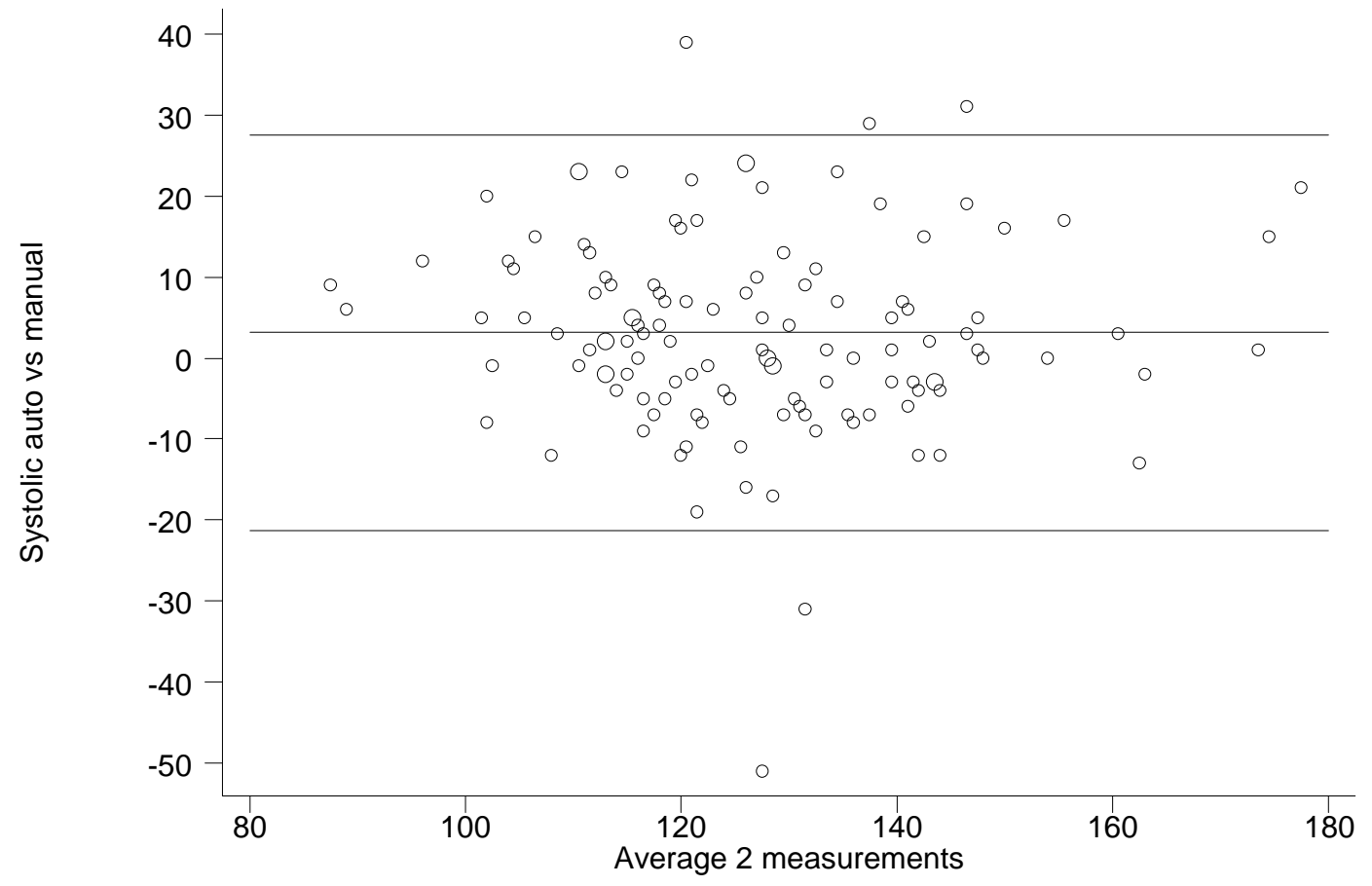

Figure 1 Bland-Altman plot for average differences in manual and automated systolic blood pressure measurements $(\mathrm{mmHg}), \mathrm{n}=126, \mathrm{r}^{2}=0.002$. 


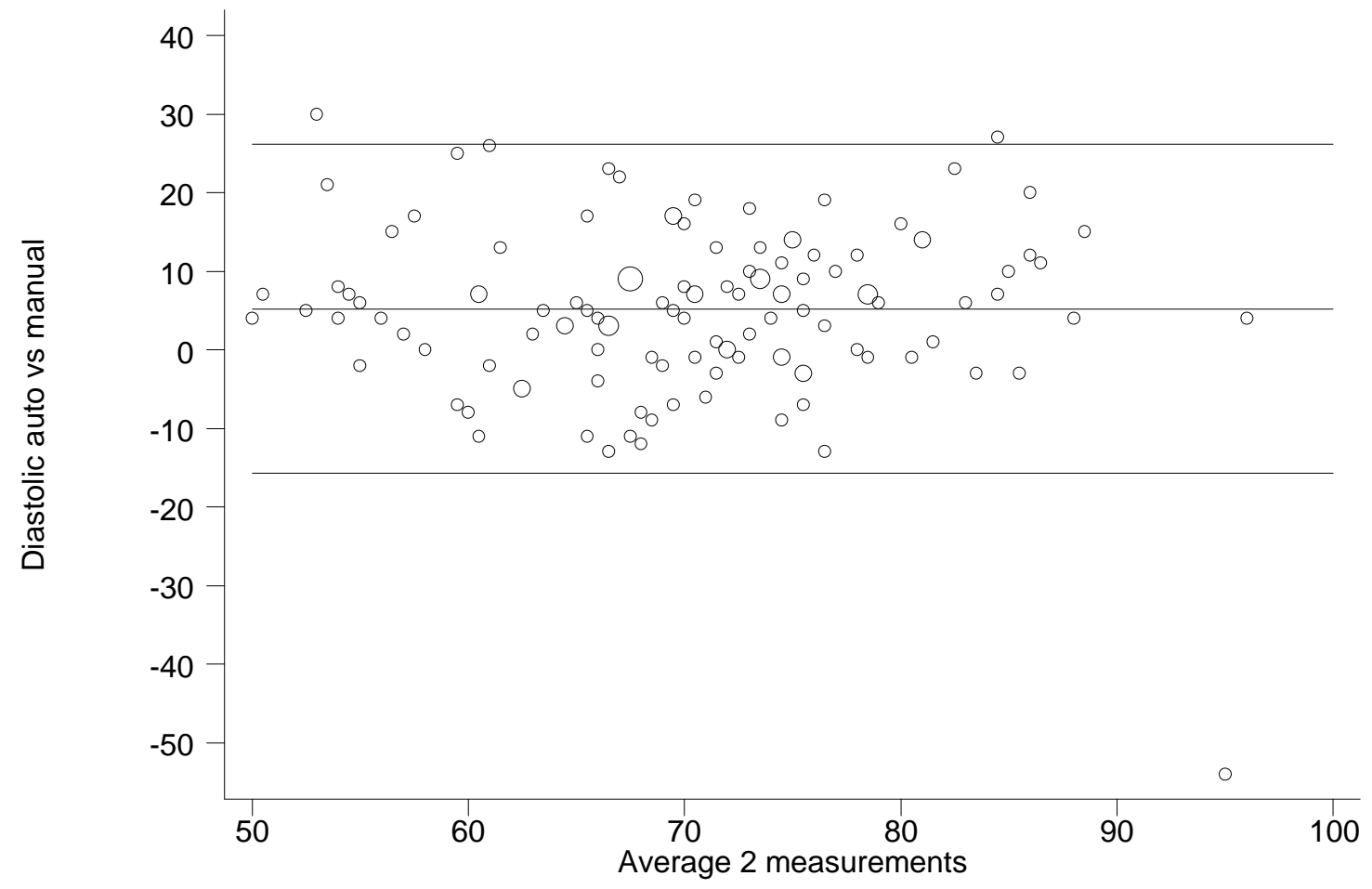

Figure 2 Bland-Altman plot for average differences in manual and automated diastolic blood pressure measurements $(\mathrm{mmHg}) . \mathrm{n}=126, \mathrm{r}^{2}=0.004$. 